\title{
Counting Quality, Not Hours: Understanding the Impact of Duty Hour Reform on Internal Medicine Residency Education
}

\author{
Diane B. Wayne, $M D^{7}$ and Karen E. Hauer, $M D^{2}$ \\ 'Department of Medicine, Northwestern University Feinberg School of Medicine, Chicago, IL, USA; ${ }^{2}$ Department of Medicine, University of \\ California San Francisco School of Medicine, San Francisco, CA, USA.
}

J Gen Intern Med 27(11):1400-1

DOI: $10.1007 / \mathrm{s} 11606-012-2185-3$

(C) Society of General Internal Medicine 2012

$\mathrm{O}$ ne year ago, the Accreditation Council for Graduate Medical Education (ACGME) imposed new resident duty hour regulations that particularly affect the most junior postgraduate learners. ${ }^{1}$ These requirements focus on enhanced supervision and oversight, and limit postgraduate year (PGY-1) residents to 16 hours of continuous duty. We expect data to emerge soon regarding the impact of the second round of duty hour reform on patient care outcomes. However, two articles in this issue of JGIM focus on the urgent need for medical educators to measure the nature and quality of resident learning and patient care activities in the context of reduced resident duty hours.

The first article, by Fletcher et al., documents the activities of PGY-1 internal medicine residents during oncall periods. ${ }^{2}$ The authors performed a prospective time and motion study on the general medicine wards of a Veterans Affairs Hospital affiliated with the Medical College of Wisconsin. Trained observers recorded activities of PGY-1 residents using customized task analysis software that divided work content into six categories: (1) direct patient care at the bedside; (2) computer work such as writing notes and orders; (3) communication with nurses or other physicians, (4) downtime; (5) transit; and (6) education and learning. Although on-call periods are assumed to be a robust opportunity for PGY-1 residents to assess and admit new patients, only $12 \%$ of PGY-1 residents' time was spent in direct patient care, as compared to $40 \%$ of time at the computer and $30 \%$ in non-patient communication. Although conferences were not included, the authors found an alarmingly small amount of time-20 min-devoted to teaching from supervising residents and/or independent reading about patients.

The second article by Gonzalo and colleagues examined compliance with 16 hours shifts during call periods. ${ }^{3}$ The authors studied the 16 hours shift in 2010 (before the 2011 mandate) at Beth Israel Deaconess Medical Center. Using an electronic survey, residents were asked about shift length and numbers of new admissions. At least one team member

Published online August 10, 2012 exceeded 16 hours during $40 \%$ of shifts. Not surprisingly, multiple admissions arriving near the end of the shift were associated with non-compliant extended shifts. However, the investigators also found that shifts with more than six total admissions per team had an adjusted odds ratio of 2.9 (95\% CI 1.05-8.3) for non-compliance. As these teams had two PGY-1 residents and a supervising PGY-2 or PGY-3 resident, this number of admissions is relatively modest, yet caused a significant amount of work hour non-compliance.

Taken together, these studies paint a sobering picture of the current status of inpatient internal medicine residency education featuring periodic on-call admitting days. Although residents have been relieved of tasks thought to be of low learning value such as blood drawing and patient transport, computerized note writing and order entry currently demand much of their time. Remaining compliant with new duty hour requirements may reduce the number of patients admitted by a PGY-1 resident to three or fewer per shift. While duty hour regulations may be intended to align PGY-1 responsibilities with learning level, current findings suggest that the timing of patient admissions, the complexity of those patients, or both, frequently preclude adherence to duty hour regulations. Structural changes to shifts to coincide with periods of higher numbers of admissions and trainee education about safe patient handoffs may help mitigate trainees' desire to stay beyond their mandated shift length to 'finish' their patients' care. Intentionally designed learning experiences that provide residents the sequence and mix of learning experiences will almost certainly prove more valuable for learning than continued reliance on historical training models that assumed that enough patient care volume would supply needed learning experiences.

We suggest a variety of potential methods to improve competency development supported by robust assessment of internal medicine residents. First, it is imperative to enhance and document the clinical skills of senior medical students, as there are fewer hours available for skill development and remediation during the PGY-1 year. Incoming residents possess a wide variety of skills, and gaps exist between what program directors expect and what trainees can actually do. ${ }^{4}$ Transition courses or student "boot camps" are a promising strategy to improve the readiness of medical school graduates to assume the patient care responsibilities of residency training. ${ }^{5}$ More broadly, 
medical school educators and program directors should collaborate to identify and implement fourth year curricular goals that transition students from the clerk to the intern role with skill in multiple competency domains. ${ }^{6}$ Competence must continue to be assessed throughout training using rigorous assessment measures. The milestones project of the American Board of Internal Medicine, in which residents acquire the knowledge, skills, and attitudes for advancing in and completing training, enhances competency-based education by setting predetermined achievement standards and also allowing individualized progression through training. ${ }^{7}$ Simulation-based education, including standardized patients, task trainers and high-fidelity models, successfully boosts residents' clinical skills to high achievement levels. ${ }^{8}$ New education models combining simulation training and predetermined achievement standards can be a powerful method to teach, assess and ensure clinical competence over time.

In addition to curricular changes, new models of clinical patient care delivery are clearly needed. Service-oriented activities that are not educational should continue to be shifted away from residents to maximize the time available for direct patient care and patient-centered learning. Attending physician supervision and teaching during evening and night shifts can also improve learning during work shifts. These models also allow for workplace-based assessment in which supervising faculty can be trained to assess residents' decision-making and patient care in real time rather than 12 24 hours after patient admissions. Daily admitting by ward teams is an effective alternative to large-volume admitting during periodic on-call periods and may potentially increase the number of patients seen and independently evaluated by PGY-1 residents. Patient logs, analogous to those used by surgical residents, can ensure adequate patient care experiences and diagnoses encountered. Schedule flexibility, perhaps in the third year, could be used to fill gaps in learning, experience or performance. Finally, rigorous medical education research is needed to help us move from counting hours to measuring quality of resident learning and work. Studies of residency redesign that include patient outcomes are particularly important.

Duty hour regulations affect not only residents but also other inpatient team members' educational experience. Medical students typically join inpatient internal medicine teams and adapt to resident schedules. Shift structures that consolidate the team's work into daytime hours, with night teams complementing them, could allow greater continuity between residents and students. However, if students see those residents spending the majority of time at computers and exceeding their mandated shift lengths, students' educational experience, and their interest in careers in internal medicine, can be compromised. Indeed, clerkship directors perceived that the 2003 resident duty hour regulations diminished students' experiences with teaching and feedback. ${ }^{9}$ As with residents, attention to the quality of students' experience through redesign of teaching experiences, supervision and patient care interactions can enhance student education. ${ }^{10}$ Attending physicians have also been affected by duty hour reform and may incur added responsibilities for patient care if resident shifts preclude their ability to provide safe, high-quality patient care. Innovative service redesign efforts that incorporate not only mandated resident requirements but also the contributions and experiences of other team members are needed.

In conclusion, two studies in this month's JGIM show that residents are seeing fewer patients on call and spending only a small percentage of their time engaged in direct patient care. Current training models are insufficient, and we must change curricula and patient care models to avoid a vicious cycle of less qualified and competent residency graduates. Rigorous multicenter studies are needed to determine best practices. As we reflect on the 2011 ACGME duty hour regulations, it is clear that it is time to stop counting resident hours and start measuring resident education quality. Clinical faculty, residency educators and medical education researchers must work together to determine effective and efficient ways to educate competent internists who deliver the highest quality patient care.

Corresponding Author: Diane B. Wayne, MD; Department of Medicine, Northwestern University Feinberg School of Medicine, Chicago, IL, USA (e-mail: Dwayne@northwestern.edu).

\section{REFERENCES}

1. ACGME Common Program Requirements. Available at: http://www.acgme.org/ acwebsite/home/Common_Program_Requirements_07012011.pdf Accessed June 30, 2012.

2. Fletcher KE, Visotcky AM, Slagle JM, Tarima S, Weinger MB, Schapira MM. The composition of intern work while on call. J Gen Intern Med. 2012. doi:10.1007/s11606-012-2120-7.

3. Gonzalo J, Herzig S, Reynolds E, Yang J. Factors associated with noncompliance during 16-hour long call shifts. J Gen Intern Med. 2012. doi:10.1007/s11606-012-2047-z.

4. Lypson ML, Frohna JG, Gruppen LD, Woolliscroft JO. Assessing residents' competencies at baseline: identifying the gaps. Acad Med. 2004;79(6):564-70.

5. Teo AR, Harleman E, O'sullivan PS, Maa J. The key role of a transition course in preparing medical students for internship. Acad Med. 2011;86 (7):860-5.

6. Lyss-Lerman P, Teherani A, Aagaard E, Loeser H, Cooke M. Harper GM. What training is needed in the fourth year of medical school? Views of residency program directors. Acad Med. 2009;84(7):823-829.

7. Green ML, Aagaard EM, Caverzagie KJ, et al. Charting the road to competence: developmental milestones for internal medicine residency training. J Grad Med Educ. 2009;1:5-20.

8. McGaghie WC, Issenberg B, Cohen ER, Wayne DB. Does simulationbased medical education with deliberate practice yield better results than traditional clinical education? A meta-analytic comparative review of the evidence. Acad Med. 2011;86:706-711.

9. Kogan JR, Pinto-Powell R, Brown LA, Hemmer P, Bellini LM, Peltier D. The impact of resident duty hours reform on the internal medicine core clerkship: results from the clerkship directors in internal medicine survey. Acad Med. 2006;81(12):1038-44.

10. Nixon LJ, Aiyer M, Durning S, et al. Educating clerkship students in the era of resident duty hour restrictions. Am J Med. 2011;124:671-676. 